$20 \mid 2012$

Des mobilités aux transports. Regards croisés en Afrique de l'ouest

\title{
Sur la frontière, quelles représentations des enfants? Enquête dans le Nord de la France
}

\author{
Xavier Leroux et Maud Verherve
}

\section{OpenEdition}

Journals

Édition électronique

URL : https://journals.openedition.org/echogeo/13057

DOI : 10.4000/echogeo. 13057

ISSN : 1963-1197

Éditeur

Pôle de recherche pour l'organisation et la diffusion de l'information géographique (CNRS UMR 8586)

Référence électronique

Xavier Leroux et Maud Verherve, "Sur la frontière, quelles représentations des enfants ? Enquête dans le Nord de la France », EchoGéo [En ligne], 20 | 2012, mis en ligne le 13 juillet 2012, consulté le 10 août 2021. URL : http://journals.openedition.org/echogeo/13057 ; DOI : https://doi.org/10.4000/echogeo. 13057

Ce document a été généré automatiquement le 10 août 2021.

EchoGéo est mis à disposition selon les termes de la licence Creative Commons Attribution - Pas d'Utilisation Commerciale - Pas de Modification 4.0 International (CC BY-NC-ND) 


\title{
Sur la frontière, quelles représentations des enfants? Enquête dans le Nord de la France
}

\author{
Xavier Leroux et Maud Verherve
}

Faire un dessin, à la fin de l'école primaire, est une activité de la pratique des arts, classée comme "grande catégorie de la création artistique ", "forme d'expression " travaillée comme medium pour le développement de la «culture humaniste ${ }^{1}$ ». Cette activité est également pratiquée en sciences physiques ou biologiques, soit pour saisir les représentations initiales des élèves avant de les faire évoluer par l'apprentissage, soit pour donner à voir le phénomène scientifique expérimenté (dessin technique ou d'observation).

En dehors de ces deux champs, la production de dessin est peu mobilisée. Si, en géographie, le dessin est utilisé depuis plus de vingt ans dans le cadre d'exercices de représentations spatiales et de réflexions sur les pratiques et images mentales (mental maps), ces expérimentations pédagogiques ne sont convoquées ni de manière générale, ni de manière fréquente. Nous avons souhaité les utiliser sur la question de la frontière, considérant avec Gumuchian (1981:39) que «le dessin constitue un matériau apte à traduire la représentation de l'espace tel que l'enfant le perçoit et s'efforce de le rendre communicable. (...) Moyen d'expression à la mesure de l'enfant, le dessin se veut signifiant et autorise une formulation plus spontanée et plus directe que l'écriture. »

C'est en 2008 que la notion de «frontière » fait son apparition dans les programmes de l'Éducation Nationale pour l'enseignement élémentaire. Depuis le tournant épistémologique de la géographie des années 1980 , c'est la première occurrence du terme dans les instructions officielles. Auparavant, si elle était présente dans les manuels scolaires que l'on a pu observer, la frontière était plutôt la charge de leçons qui devaient justifier l'État et ses limites ${ }^{2}$. Après deux ans d'application, nous avons voulu voir si et comment les élèves se représentent la frontière, dans une région où elle est devenue interface. 
Une étude a donc été menée pour recueillir des témoignages d'enfants du Nord de la France sur la frontière, et comparer leurs paroles et leurs dessins afin de répondre à notre interrogation : quelle géographie de la frontière peut se faire un enfant qui habite lui même dans une région frontalière ? Comment se construit la représentation de la frontière chez des enfants de 8 à 11 ans, auxquels l'école doit enseigner cette notion alors même qu'ils peuvent la vivre dans leur quotidien?

\section{Faire parler les enfants : des expériences, des mots, un dessin}

Nous avons mené une enquête d'environ 6 mois au début de l'année 2010 dans des classes de cycle 3 volontaires (de $\mathrm{CE} 2$ à $\mathrm{CM} 2$ ) $^{3}$. Matériellement, il ne nous était pas possible de nous livrer à une étude quantitative permettant d'examiner si les représentations variaient en fonction de l'éloignement de la frontière belge, ou selon la proximité de la Belgique francophone ou néerlandophone. Nous avons en effet diffusé le questionnaire (voir annexe ) dans notre réseau personnel et par l'intermédiaire d'inspecteurs ayant accepté de le relayer. Plus d'une cinquantaine d'enseignants du département $d u$ Nord nous ont répondu. A raison d'une moyenne de 25 élèves par classe, ce sont quelques 1200 représentations qui ont donc été recueillies.

Ce questionnaire se terminait par un dessin, outil qui semblait adapté pour compléter les réponses rédigées et permettre aux élèves en difficulté à l'écrit de s'exprimer autrement. En effet, ce type de questionnaire sur papier peut révéler, parfois, une difficulté face au monde de l'écrit. Aussi, quelques questions sont restées sans réponse. Ce dessin est donc devenu, au cours de l'analyse, le matériau révélateur de la distance entre les représentations scolaires de l'élève et celles de son vécu d'enfant.

Les enseignants avaient pour consigne de ne pas préparer ni expliquer le questionnaire de manière à recueillir, auprès de leurs élèves, les visions les plus spontanées possibles. Il était simplement présenté à l'enseignant en spécifiant le contexte de l'enquête, dégagé des objectifs d'apprentissage. Pour pouvoir saisir les représentations initiales des élèves, il était en effet nécessaire d'insister sur l'aspect "décroché" de cette activité (« ce n'est pas noté ", « ça ne compte pas pour le bulletin », « ce n'est pas pour l'école»), ne comptant pas dans leur travail quotidien et ne faisant pas intervenir le maître.

L'élaboration d'une méthodologie d'enquête auprès de jeunes enfants est une problématique de recherche en elle-même ${ }^{4}$. Le questionnaire a été composé de manière à alterner les questions ouvertes et fermées, proposer des formulations suffisamment claires pour être comprises mais avec un sens suffisamment large pour récolter des témoignages solides et authentiques.

En cela, notre démarche expérimente une méthodologie élaborée à partir de réflexions et de choix sur :

- l'énonciation des questions :

Pour savoir si les élèves percevaient des marqueurs paysagers du franchissement de la frontière (signalétique, institution, contrôle...), nous leur avons demandé ainsi : « As-tu vu quelque chose de particulier avant de rentrer dans un pays et si oui, de quoi s'agissait-t-il ? ». En cela, cette question est restée la plus abstraite de notre enquête. Si $50 \%$ des enfants répondent «non », 30 \% justifient leur réponse affirmative sans rapport avec le seuil 
frontalier lui-même, mais plutôt avec des spécificités culturelles du pays en général (la langue, l'alimentation...). $20 \%$ seulement proposent une réponse pertinente relative à la signalétique (panneaux d'entrée dans le pays, drapeaux) ou l'institution (policiers, douaniers).

- l'ordre des questions :

Poser en ouverture de questionnaire « Es-tu déjà allé dans un autre pays que la France ? Si oui, le(s)quel(s) ? » visait à apprécier le degré de mobilité chez les élèves mais, surtout à voir si la Belgique leur venait spontanément à l'esprit. Elle n'a été citée ici, qu'à hauteur de $30 \%$ des réponses. Plus loin dans l'enquête, plus de $70 \%$ ont répondu par l'affirmative à la question de savoir s'ils étaient déjà allés en Belgique. Ce développement spiralaire du questionnaire devait donc permettre de nuancer les résultats et ne pas orienter les élèves vers des réponses attendues ${ }^{5}$.

- le matériau des représentations recueillies :

Après cinq questions faites pour obtenir leurs témoignages de passage de frontière (francobelge ou autres), les élèves ont été invités à livrer deux types de représentations, langagière d'une part et iconographique d'autre part. Ainsi pouvaient-ils d'abord nous donner cinq mots (maximum) « leur évoquant la frontière » et ensuite, le dessin de « l'image qu'ils avaient dans la tête » en pensant à ce terme.

\section{Des images de la séparation, des témoignages de l'interface}

L'examen des dessins permet de structurer l'analyse des représentations par confrontation et comparaison. $80 \%$ d'entre eux représentent des frontières variées : cartes, paysages, scènes; non localisées, franco-belges, situées ailleurs. Mais que dire des $20 \%$ restants ? Ils révèlent, et ce pour tous les niveaux, une absence totale d'image de la notion de frontière chez les élèves. Beaucoup de dessins, en effet, soit donnent à voir ce que l'enfant avait envie de dessiner à ce moment-là sans rapport avec le questionnaire, soit associent le mot frontière à un mot connu dont le son est proche (front du visage, fontaine).

Ces dessins ne sont pas seuls à révéler le manque de représentation des frontières. La présence récurrente de dessins de fontaine nous a ainsi fortement surpris. En parallèle, «fontaine » arrive en $12^{\mathrm{e}}$ position des mots recensés, sur le classement de 80 termes. Les enfants qui citent le mot «fontaine » sont donc cohérents dans leur représentation, aussi erronée soit-elle, puisqu'ils la redoublent en la dessinant. Ce résultat, obtenu majoritairement auprès d'élèves de CE2 (qui débutent leur apprentissage en géographie), confirme un déficit dans la représentation si important que le mot «frontière » n'est pas associé à un contexte géographique, alors même qu'ils sont capables de témoigner d'expériences de passages de frontière.

\section{Les « cartographies »}

Par les représentations de type cartographique, c'est le langage traditionnel de la frontière, ligne séparatrice entre deux pays, qui s'exprime.

Le dessin réalisé par Lucas (illustration 1), est emblématique de ceux que l'on a pu rassembler dans cette catégorie qui traduit souvent l'espace européen. Ce rapprochement entre frontière et espace de l'Europe, essentiellement opéré par des 
classes de $\mathrm{CM} 1$ et $\mathrm{CM} 2$, s'explique logiquement par des programmes qui requièrent d'étudier « les frontières de l'Europe ", en particulier pour les classes de CM1. Ce genre d'image correspond donc à l'enseignement reçu par l'élève qui travaille sur l'Europe à partir de l'étude de sa mosaïque cartographique et peut ensuite attester de sa compréhension de l'espace européen par la restitution de son découpage étatique. La reproduction la plus fidèle signifie donc qu'on a retenu sa leçon. L'élève peut percevoir dans cet exercice cartographique le modèle même de l'activité géographique, et apprécier de composer ce puzzle sur papier dont les pièces sont autant de pays à colorier.

Ici, la capacité d'abstraction nécessaire à comprendre ce que ces pièces signifient comme territoires n'est pas vérifiée chez l'élève ${ }^{6}$. Ces représentations constituent donc, d'après nos données, le modèle des frontières construit chez les élèves. Les enjeux politiques de leurs fonctions ou leurs formes ne sont pas perceptibles ni mis en jeu dans ce type de représentation.

Illustration 1 - Dessin de Lucas, 11 ans, CM2, Roubaix

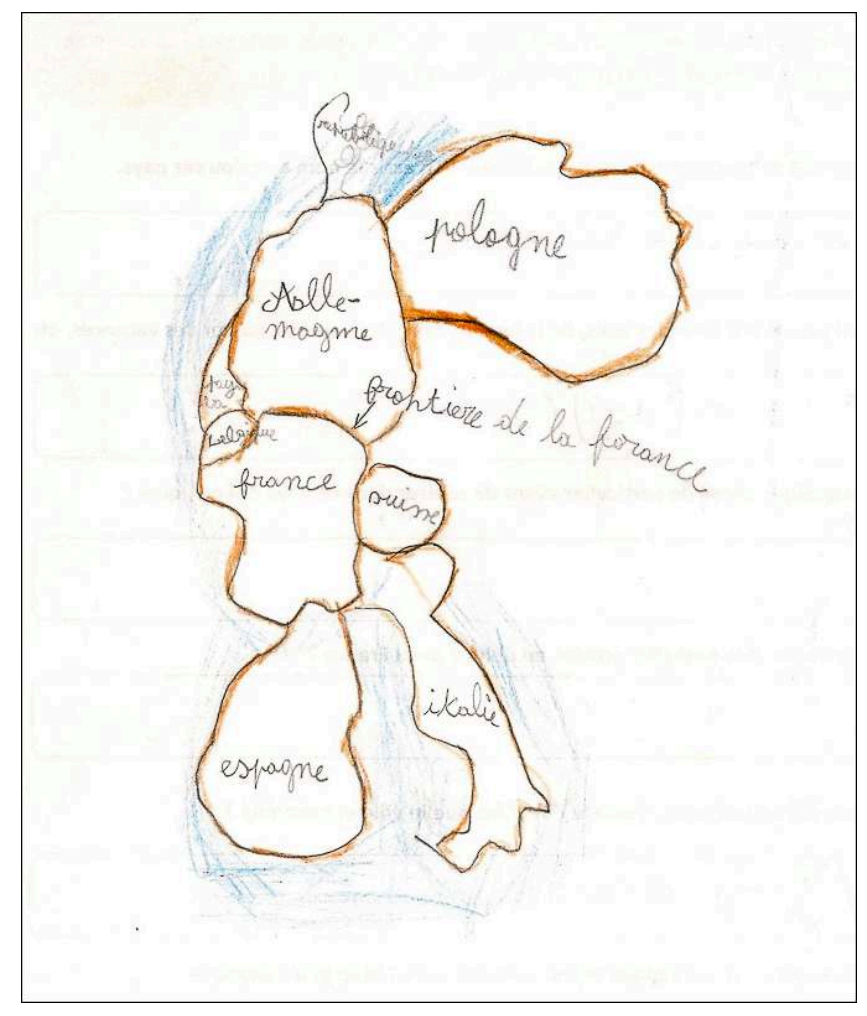

La frontière est toujours, à cette échelle, une ligne continue qui marque la distinction : le rôle de la frontière, qui pourrait être symbolisé par une ligne discontinue ou des jeux de flèches, n'affine la perception dans aucun dessin. Le dessin réalisé par Clémentine (illustration 2) représente la définition de la frontière la plus abstraite possible, que le dessin de Marvin (illustration 3) prolonge en spécifiant les différences, apprises en classe, entre frontières terrestres et frontières maritimes. 
Illustration 2 - Dessin de Clémentine, 10 ans, CM2, Bailleul

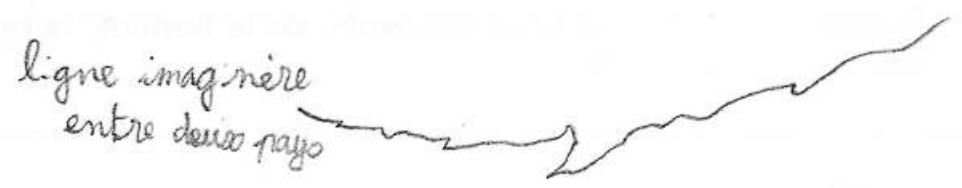

Illustration 3 - Dessin de Marvin, 11 ans, CM2, Colleret

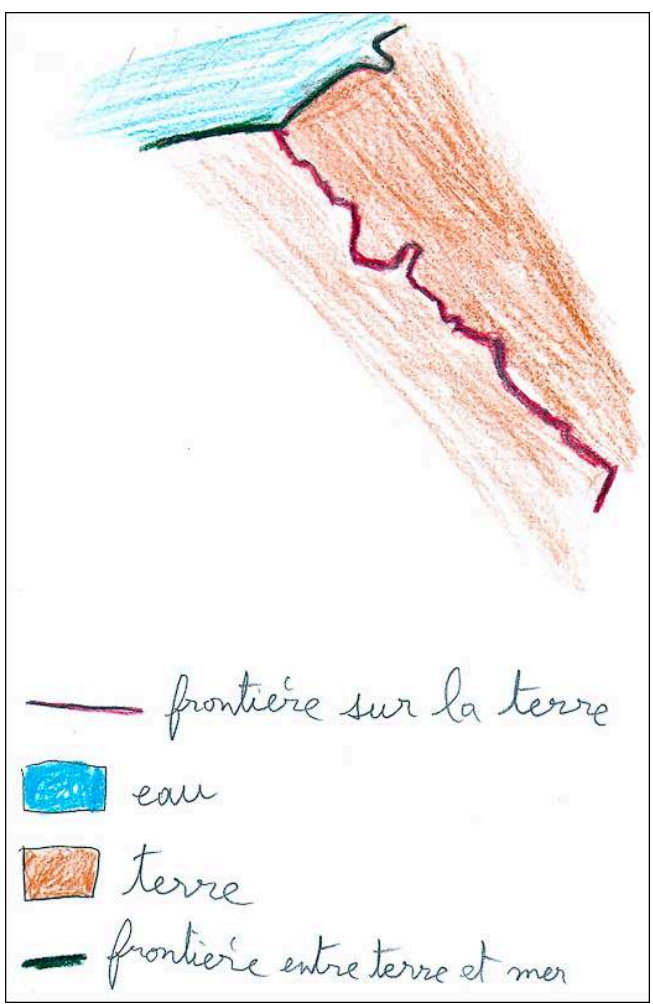

Même entre la France et la Belgique, le dessin réalisé par Louisa (illustration 4) montre bien la force de cette frontière barrière que se représente l'élève, plus fortement marquée (par les croix) que les coutures intérieures de l'espace administratif français. Et pourtant Louisa témoigne d'une expérience régulière de la Belgique et ne la considère pas comme un pays étranger du fait d'une moitié francophone. 
Illustration 4 - Dessin de Louisa, 10 ans, CM2, Emmerin

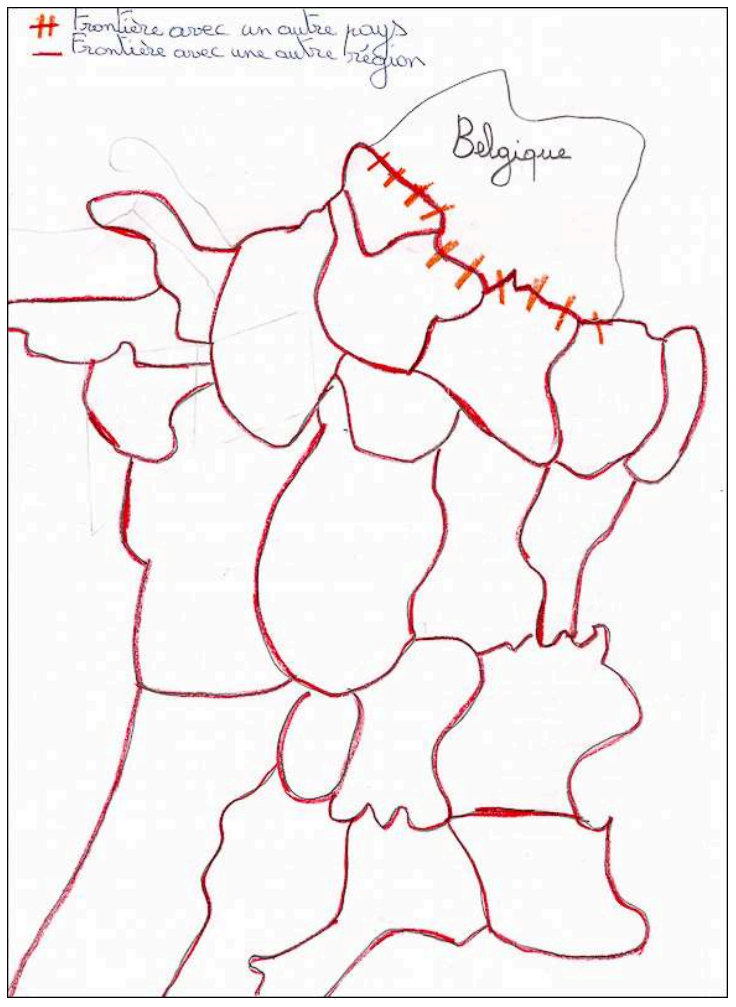

Utilisée sans nuances, la cartographie apparaît, au regard de ces dessins, comme un exercice simplifié et caricaturé chez les élèves, voire comme un exercice simplificateur et caricatural dans la pratique des classes.

\section{Frontière et barrières naturelles}

Cette vision traditionnelle de la frontière héritée comme ligne séparatrice est voisine d'une autre représentation, issue de la géographie classique : qu'ils soient localisés ou non, en effet, nombreux sont les dessins de frontières naturelles, comme si la nature, avant tout, structurait les frontières. Le dessin produit par Nicolas (illustration 5) est emblématique. 


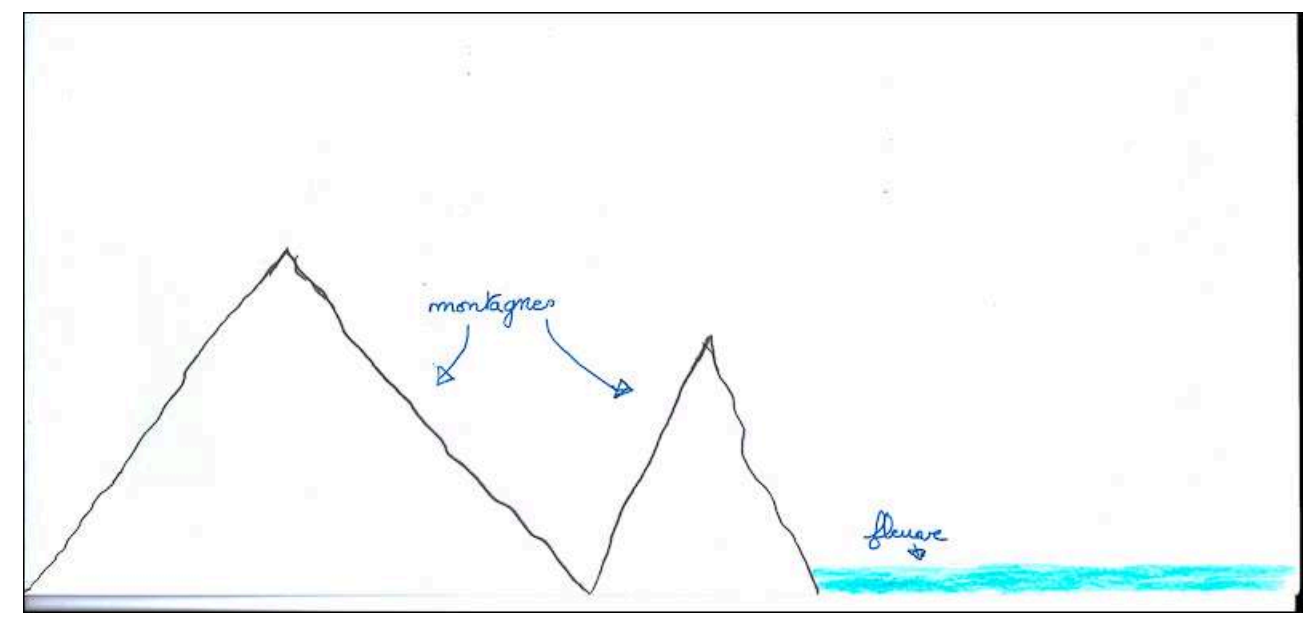

L'empreinte laissée par les manuels scolaires qui introduisent encore et toujours la frontière par des photographies d'un sommet alpin ou pyrénéen, voire de quelques mètres d'un fleuve (Oyapock, Rhin), que seule la légende permet d'identifier comme tels, est palpable, dans ce type de dessins. Ces imageries scolaires véhiculées par certains manuels construisent une représentation simplifiée de la frontière par la barrière naturelle. Le témoignage de Nicolas va dans ce sens puisqu'à la question « astu vu quelque chose de particulier avant de rentrer dans un autre pays? », l'enfant répond qu'il a vu des montagnes avant d'entrer en Italie mais rien de particulier avant d'entrer en Belgique.

Ces images sont réellement intégrées puisque les éléments naturels, comme rivière, fleuve, océan/mer, montagne ou encore lac, arrivent aussi en tête de liste des mots recensés dans le questionnaire avec. Sans doute que "mer", "fleuves" et "montagnes" ont pu marquer les expériences géographiques des enfants (vécues lors des vacances familiales en France ou dans les pays d'origine, par-delà la Méditerranée) comme en témoignent de nombreux dessins à l'image de celui de Naïma (illustration 6). 


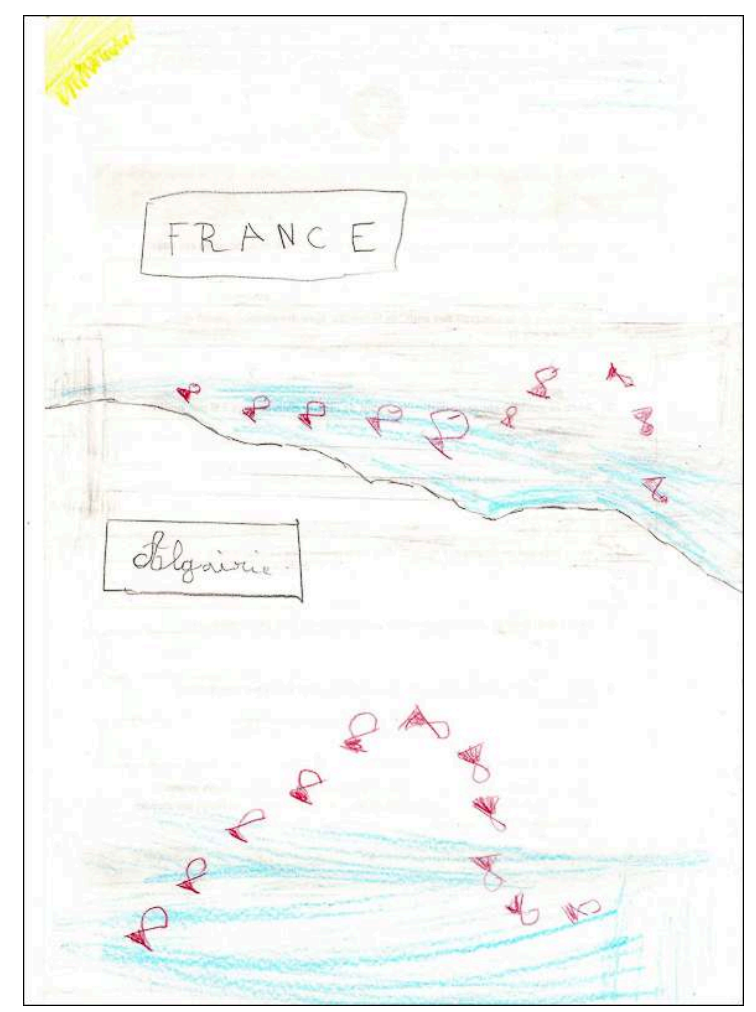

On peut penser que les enfants vivent le franchissement de la mer Méditerranée ou des Pyrénées comme des discontinuités marquées par une séparation spatio-temporelle (celle d'avec leur paysage habituel, celle d'avec leur quotidien). Par suite, si cette fonction de séparation détermine pour eux exclusivement la frontière ${ }^{7}$, alors, ils peuvent assimiler fonction de séparation et sens de la frontière, et finalement penser que toute frontière est séparation, toute séparation frontière.

Pour renforcer cette idée, les bordures de la France que sont le Rhin, les Alpes, les Pyrénées sont enseignées comme les frontières emblématiques du pays, et souvent retenues dans les esprits comme modèle naturel de la frontière. Précisément, c'est cette généralisation du cas particulier à l'archétype qui pose problème. La très fréquente occurrence du mot "lac ", et les dessins très nombreux le représentant, confirment encore cette idée car, pour les frontières françaises, seul le cas du lac Léman fonctionne.

L'imaginaire collectif hérité des traditions de la géographie classique se révèle au travers des représentations et prend un tour plus politique encore dans les dessins d'une frontière à l'échelle « continentale ». 


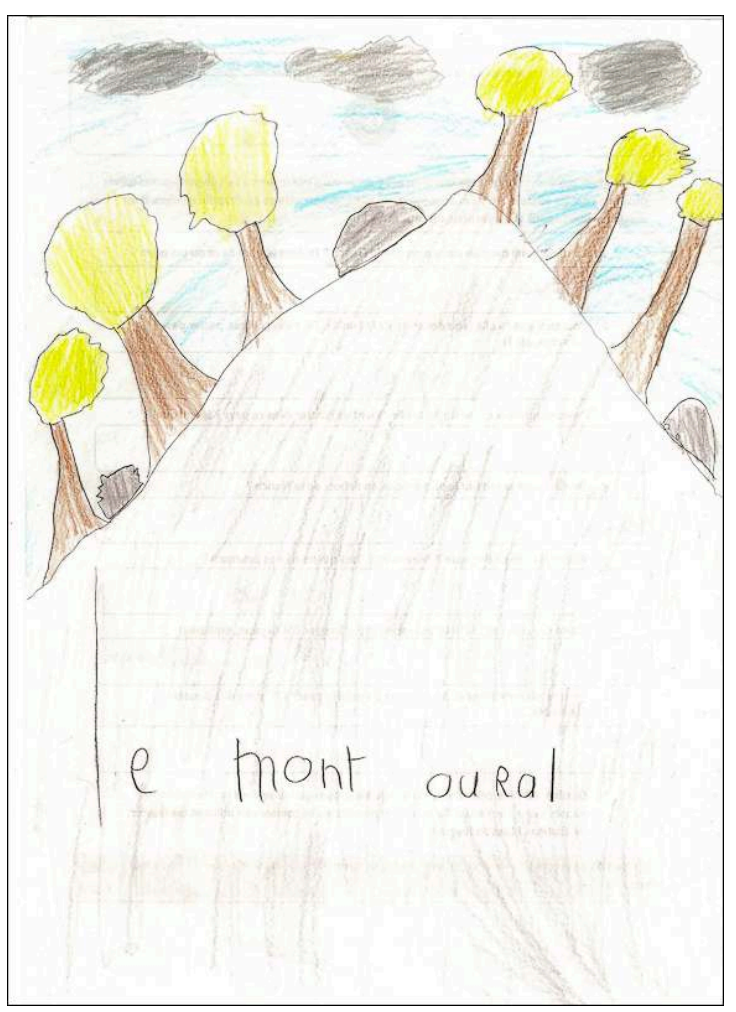

Le dessin réalisé par Rania (illustration 7), témoigne bien, en effet, du type d'image d'Epinal qui résiste dans l'enseignement. Ici, par cette représentation enfantine d'un paysage symbolique de la chaîne de l'Oural, nous sommes emmenés sur les anciens confins imaginaires de l'Europe, marqueurs d'une certaine vision du monde plus que d'une discontinuité spatiale - l'Oural ne représentant pas un obstacle au franchissement.

\section{Frontière et fonctions de contrôle}

La frontière apparaît également, dans les mots comme sur les dessins, par certaines de ses fonctions de contrôle. Les mots associés à la frontière par les élèves sont ici : barrière, barrage, mur, muraille, police, policier et surtout douane et péage. L'emploi de ces termes peut signifier l'intégration par les élèves de certaines images médiatiques des frontières, relayant les contrôles d'identités ou les saisies de drogues. Les franchissements contrôlés des frontières, qui représentent une partie de leur expérience loin du quotidien, donnent tout le sens, pour eux, à la frontière définitivement conçue comme séparation (à l'inverse de l'expérience régulière de passage libre en Belgique dont ils témoignent comme nous le verrons). La confusion entre la douane et le péage renforce cette ambiguïté entre fonction et sens de la frontière. Pour eux, un temps de pause, la présentation d'un document à une personne et le lever de barrière, c'est passer une frontière, qu'importe qu'on présente des papiers d'identité ou qu'on prenne et paie un ticket de péage comme le montrent les dessins réalisés par Jordan et Jessica (illustrations 8 et 9). 
Illustration 8 - Dessin de Jordan, 9 ans, CM1, Tourcoing

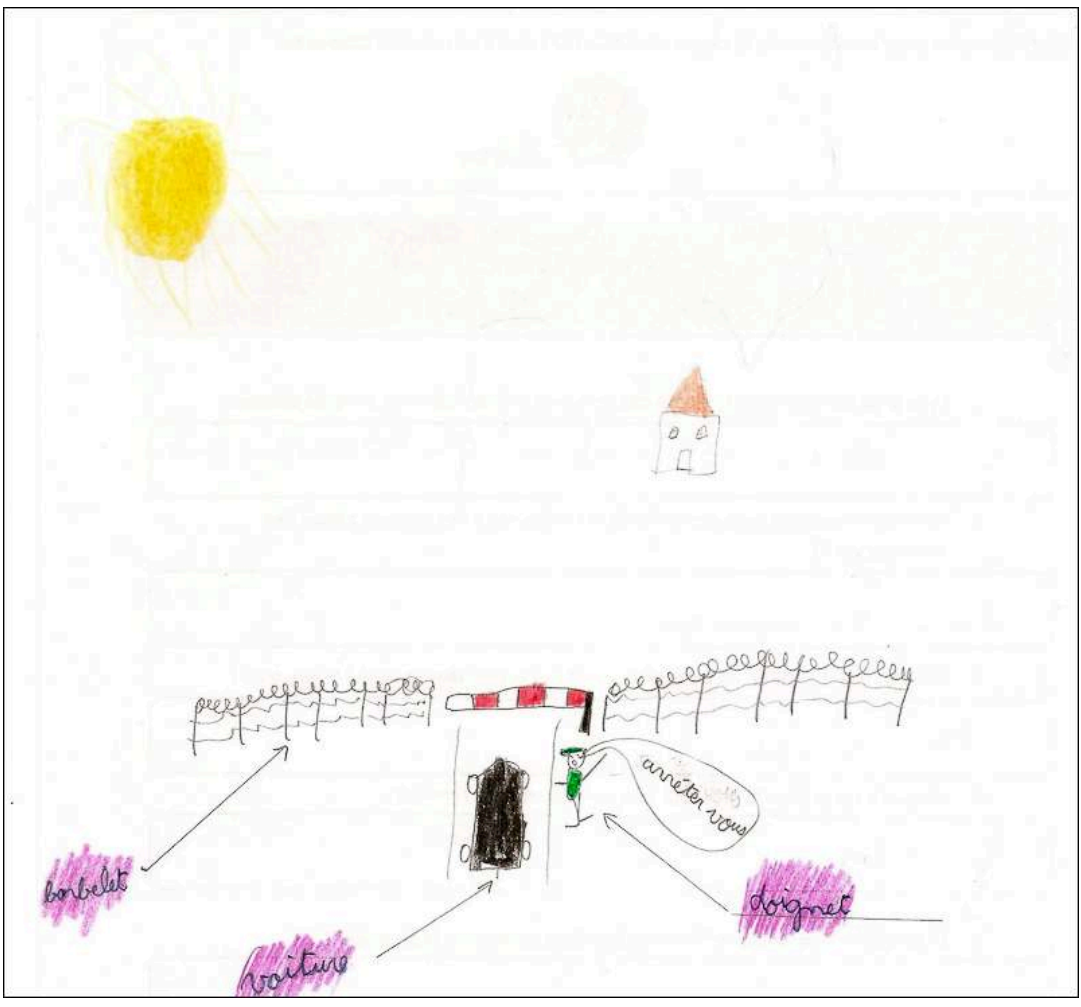

Illustration 9 - Dessin de Jessica, 10 ans, CM2, Maroilles

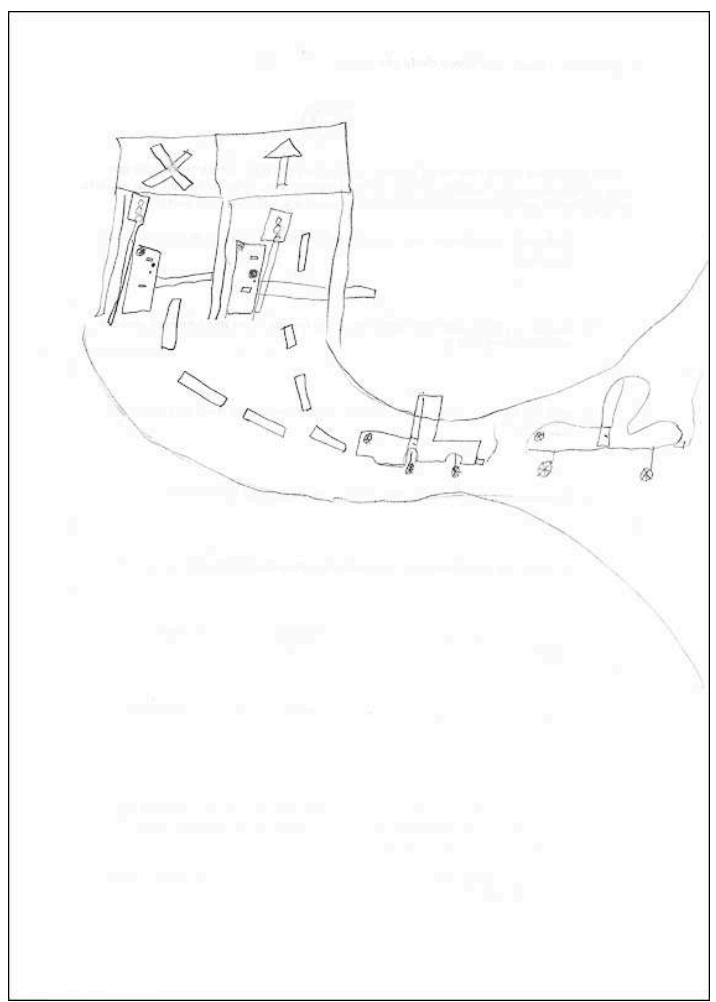

C'est bien que la fonction politique de contrôle, en jeu aux frontières, n'est pas identifiée. En conséquence, aucune spécificité liée à la situation géographique des enfants qui vivent régulièrement et témoignent des relations transfrontalières entre la 
France et la Belgique ne se dégage de ces représentations. Les dessins restent proches de stéréotypes, de visions simplifiées ou caricaturales, et reposent sur des expériences qui n'impliquent pas leur pratique régulière de la frontière.

$\mathrm{Au}$ plan politique, on trouve parfois une extension de l'idée de ligne séparatrice dans quelques dessins, avec une vision géopolitique de la frontière, structurée par les murs et les conflits armés, comme le manifeste le dessin de Fanny (illustration 10) qui indique être allée au Congo lorsqu'elle était plus petite sans qu'il soit possible de vérifier qu'elle y ait assisté à un conflit :

Illustration 10 - Dessin de Fanny, 8 ans, CM2, Roubaix

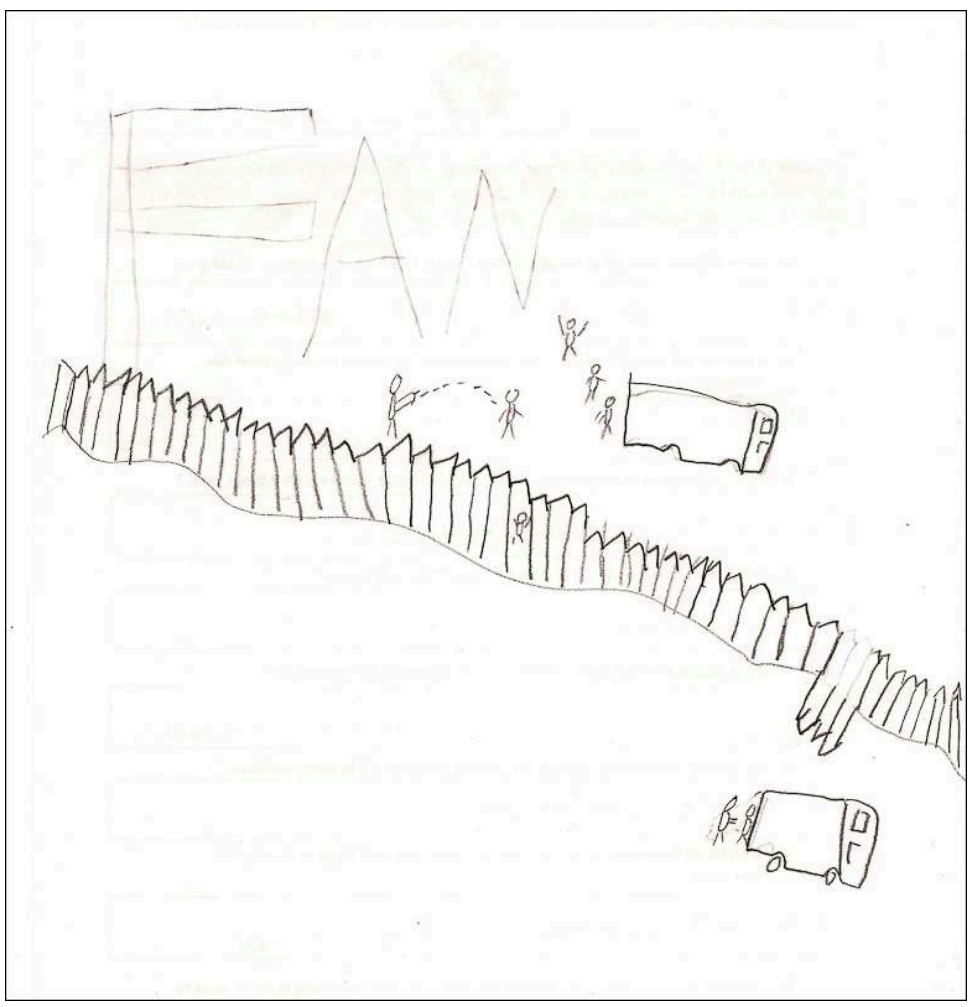

Toutefois les représentations de murs et barbelés sont rares et on ne parvient pas à déceler le rôle de l'école dans celles-ci, alors même que ces objets, très actuels dans la recherche, rendent visibles et lisibles certaines problématiques complexes des frontières aujourd'hui. Dans la plupart des représentations, le rôle politique et humain des frontières, lié aux flux migratoires et aux récits de vie qui les accompagnent, est absent

\section{Frontière, ailleurs, altérité}

Certains dessins cristalisent cette représentation de l'outre-frontière comme de l'étranger en symbolisant les différences culturelles. 
Illustration 11 - Dessin de Adil, 11 ans, CM2, Tourcoing

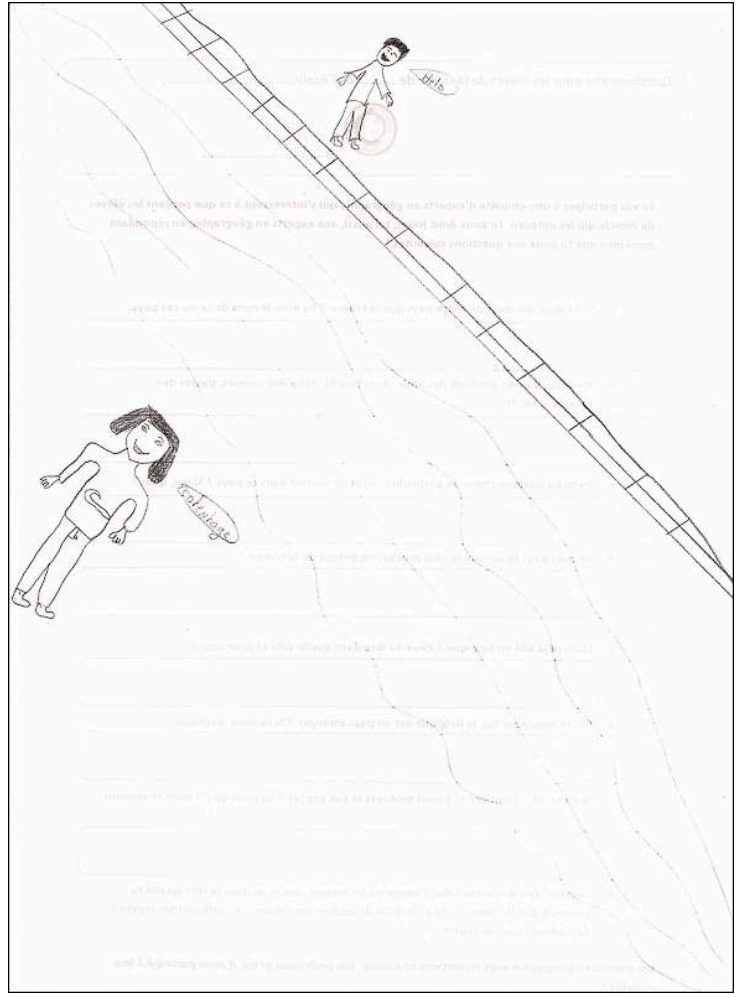

Illustration 12 - Dessin de Gwendoline, 9 ans, CM1, Douai

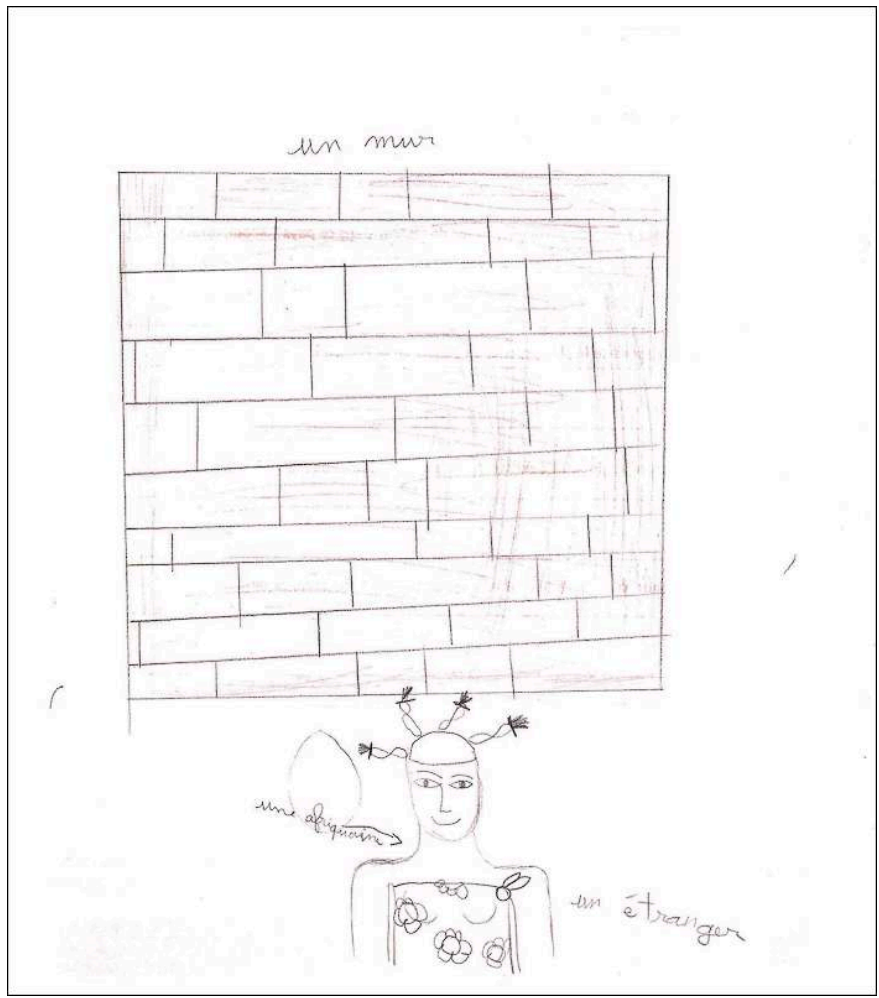

«Fenêtre souvent déformante sur l'Ailleurs et l'Autre » (Renard, Picouet, $2007: 30)^{8}$, à l'école la frontière est une notion qui se dissout dans des discours humanistes aux perspectives pluridisciplinaires, prise dans son registre symbolique. Adil (illustration 
11) dessine un anglophone et un germanophone de part et d'autre d'un mur qui n'a pas, a priori, de réalité géographique possible. On suppose que l'élève a mobilisé les deux langues qu'il connaît- les plus étudiées à l'école primaire dans le Nord- et a représenté deux individus symboliques, en quelque sorte déterritorialisés. Gwendoline (illustration 12), quant à elle, dessine une "africaine " souriante comme l'image de cette altérité positive, représentante du cosmopolitisme que l'école valorise dans de nombreux projets citoyens. Toutefois, une tension présente dans les dessins de ces deux élèves vient fragiliser l'idéologie d'un monde sans frontières: la présence du mur dans les deux dessins, et le redoublement du mot «africaine » par «étranger» dans celui de Gwendoline. L'Ailleurs et l'Autre représentent alors exotisme, attrait du lointain et en même temps peur de l'inconnu et repli.

\section{Expériences des frontières}

C'est bien la distance qui existe entre ces représentations de la frontière et l'expérience qu'en ont ces enfants dans leur vie quotidienne que révèle le rapprochement de leurs mots, dessins et témoignages.

Le franchissement de la frontière vers la Belgique apparaît en effet dans la majorité des témoignages des élèves. Lorsqu'on leur demande s'ils sont déjà allés en Belgique, nous l'avons dit, 70 \% des élèves en attestent. Il se peut même que ce chiffre soit plus élevé encore en réalité : les enfants résidant à Tourcoing, par exemple, vont faire des courses à Mouscron en suivant un tel continuum urbain qu'ils ne changent ni de trottoir, ni de nom de rue. Ainsi, le dessin de Matéo (illustration 13), montre une forte appropriation du territoire voisin sous la forme d'un continuum, ce qu'il confirme en expliquant qu'il se rend fréquemment en Belgique pour y faire des courses ou aller voir de la famille.

Illustration 13 - Dessin de Matéo, 10 ans, CM2, Tourcoing

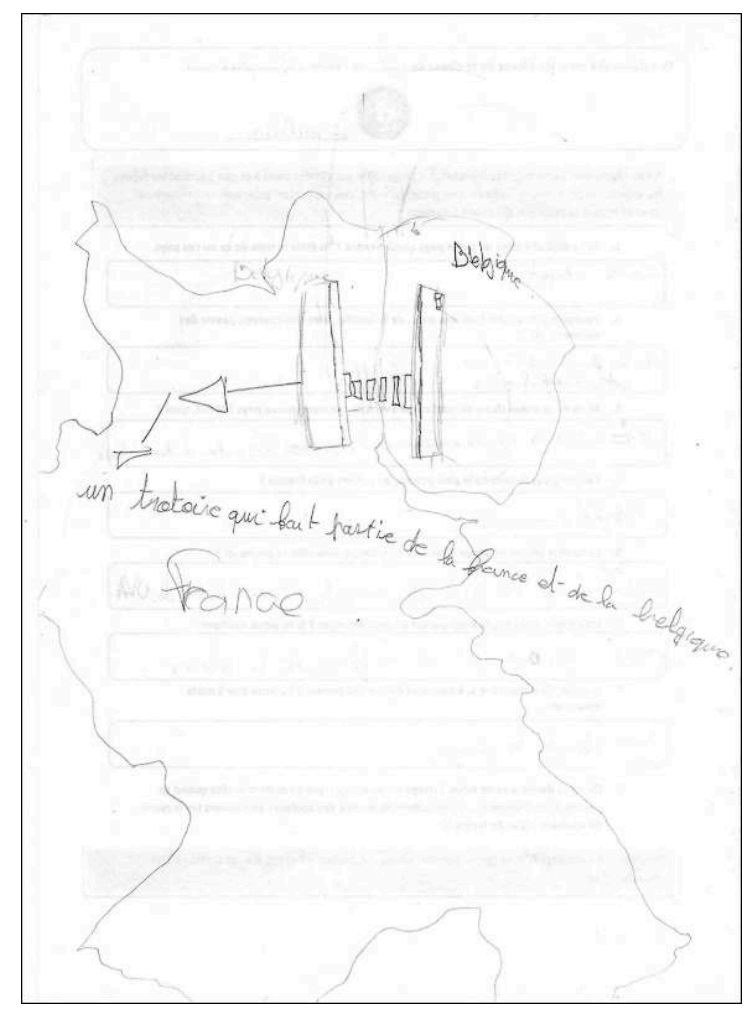

EchoGéo, 20 | 2012 
La Belgique, dans ce cas, est davantage l'autre bout de la rue qu'un autre pays que la France. La conscience du franchissement n'est donc pas toujours évidente chez les élèves puisque c'est d'abord de la continuité dont ils témoignent alors qu'ils identifient, comme nous l'avons montré, la frontière quand elle est séparation. Ils se sentent d'ailleurs proches de la Belgique plus que d'un autre pays (50\% des réponses à la question «de quel pays te sens tu le plus proche en dehors de la France?») et considèrent même, à $70 \%$, qu'elle ne constitue pas un pays étranger (à l'aide de justifications multiples : «j'y vais souvent ", « on parle la même langue ", « on a la même monnaie »...). A propos du bassin genevois, André (1989) notait que, sur 500 dessins, la frontière franco-suisse ne figurait pas sur les $3 / 4$ d'entre eux, témoignant non d'un oubli mais de "l'affirmation d'un espace homogène franco-suisse ». De la même façon, quoique de manière limitée, on voit émerger l'affirmation d'un espace homogène franco-belge. Lorsqu'on a ainsi demandé à Ludovic (illustration 14) de dessiner l'image qu'il avait dans la tête quand on lui dit le mot « frontière », il a représenté la France et la Belgique de deux couleurs distinctes et séparées par une ligne continue, mais uniquement la France et la Belgique, dans des proportions équivalentes, et côte à côte. Le dessin traduit donc l'idée que les deux pays, si proches des expériences de Ludovic, doivent avoir le même poids et donne bien à voir une homogénéité franco-belge. Plus nettement encore, le dessin de Jeanne (illustration 15) a complètement omis de représenter la Belgique (alors qu'elle l'évoque dans ses expériences), ce qui pourrait signifier que pour elle, la Belgique est intégrée au Nord de la France. Dans les réponses au questionnaire écrit, ces deux élèves ne considèrent pas la Belgique comme un pays étranger.

Illustration 14 - Dessin de Ludovic, 10 ans, CM2, Lille

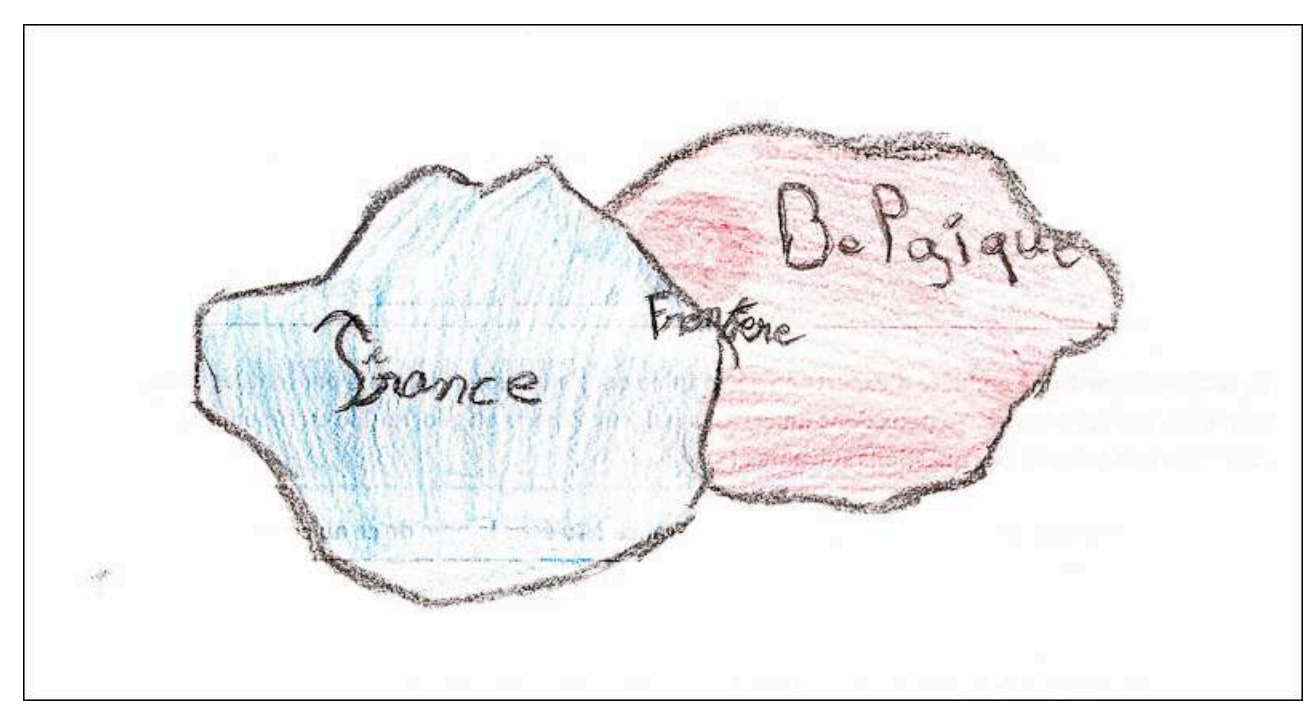




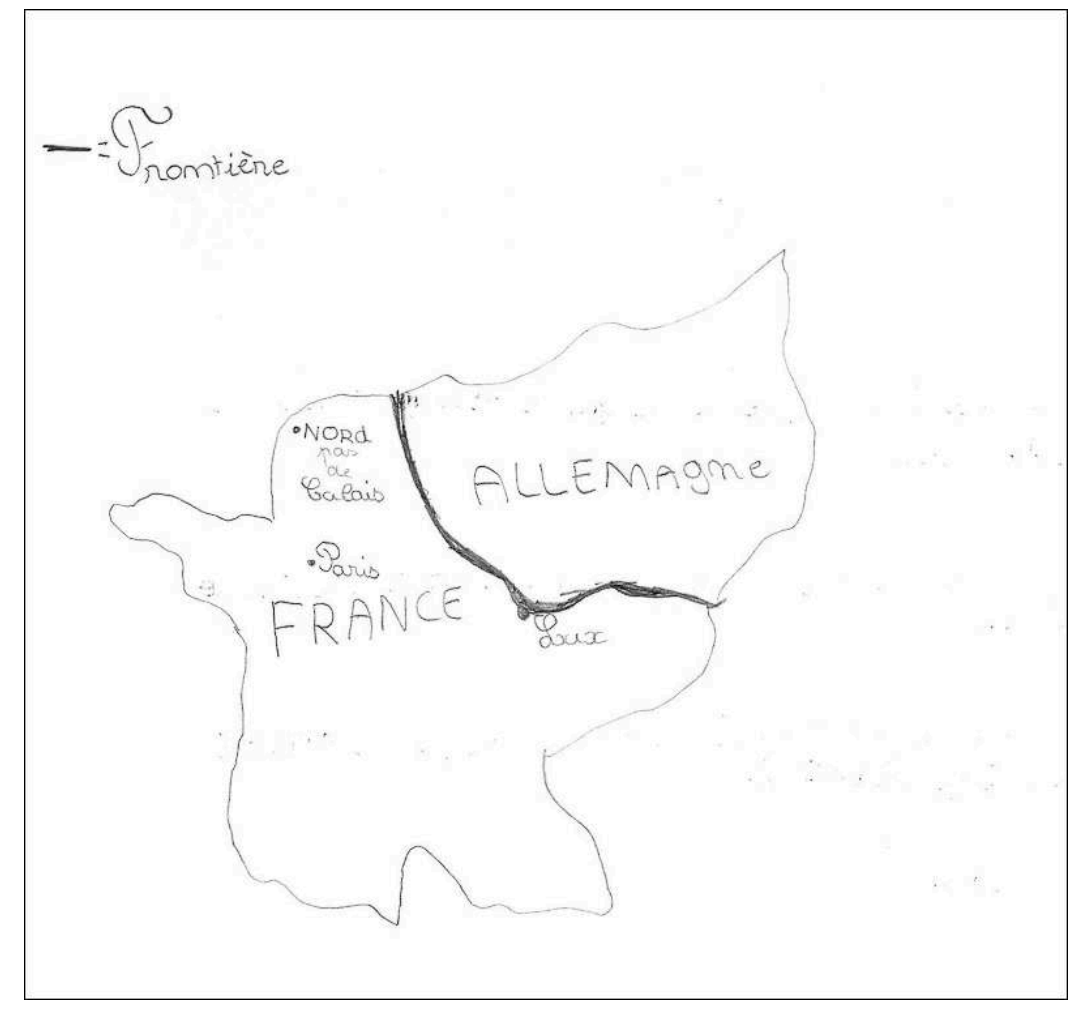

Cette réalité franco-belge dont témoignent ces dessins se traduit encore dans les paroles des enfants sur les raisons pour lesquelles ils vont en Belgique. Dans la pratique, les motifs commandant aux déplacements en Belgique tiennent beaucoup à l'agrément (visites à la famille, parcs d'attractions) ou à l'achat de produits ciblés (essence, chocolats, alcool, tabac, ou frites). On retrouve les clichés d'une frontière exotique et d'une frontière socio-économique (Crozat, 2001)

En conséquence, ces témoignages de passage vers la Belgique ne sont pas traduits par les mots et dessins de frontières, puisque les enfants témoignent d'un vécu quotidien, $\mathrm{y}$ compris ceux qui ne parviennent pas à en donner de représentation probante. Les expériences récurrentes des enfants les lient, dans leur pratique spatiale, à la Belgique. Leurs circulations dans ce pays, leurs motivations, leurs habitudes montrent ainsi qu'ils connaissent, sans le savoir, la frontière dans le registre de l'interface. La ligne continue séparatrice de leur carte scolaire dessine de fait, pour eux, un trait discontinu, laissant opérer échanges et relations. Or, il n'y aurait aucune difficulté à faire saisir aux élèves que la porosité de la frontière qu'ils vivent dans leur région se traduit par cette ligne discontinue, qui ne sépare pas, et qui pourtant s'appelle frontière. Ainsi, sur ces territoires frontaliers du Nord, l'enseignement de la notion de « frontière de l'Europe " ne permet pas de faire comprendre la portée de l'expérience géographique des enfants, en ne faisant rien de la riche complexité du local. Pourtant une question du programme permettrait de valoriser ce vécu, de ne pas entraîner les élèves dans une pratique caricaturale de la cartographie, et de donner sens, à une autre échelle surtout, aux dynamiques spatiales qu'ils vivent. En effet, les programmes de 2008 réclament de faire du local un véritable champ d'étude pour le cycle 3. C'est une nouveauté pour ces instructions officielles que de traiter « des réalités géographiques locales à la région où vivent les élèves ». 
Quelques rares exceptions, dans ce questionnaire, accréditent cette idée. Le dessin de Clara (illustration 16) en est une illustration puisqu'elle a ici symbolisé la limite de sa commune et non la limite avec l'État voisin, preuve que c'est à cette échelle locale qu'elle se représente les choses et ce, sans changer de couleur ou de symboles, comme pour montrer qu'il n'y a pas de réelle discontinuité.

Illustration 16 - Dessin de Clara, 11 ans, CM2, Willems

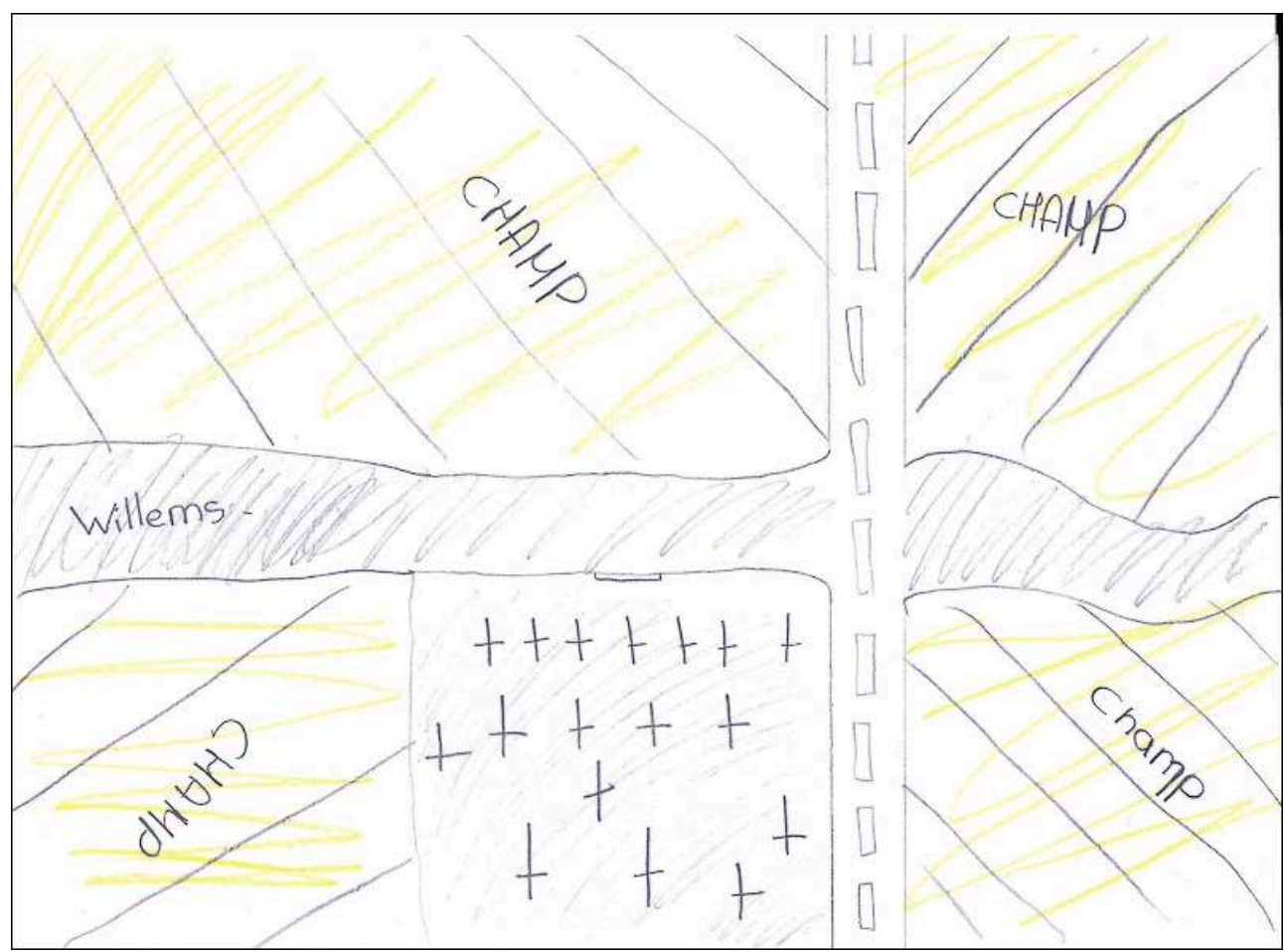

Ce dernier dessin montre bien qu'il est possible de construire des représentations du transfrontalier pour des élèves de primaire -en région frontalière du moins. Dans le cas $\mathrm{du}$ Nord, enseigner les frontières de l'Europe devrait donc pouvoir passer par le local, pour faire comprendre aux élèves, précisément, l'importance des politiques européennes dans leur quotidien et où, sans qu'ils le sachent, certains effets-frontière jouent. Loin de réduire le local à la proximité (la rue, le quartier, la commune), il est possible de l'utiliser pour rendre compte de la complexité d'une frontière où se jouent des réalités pluriscalaires ${ }^{9}$ et faire évoluer en ce sens les représentations d'un objet spatial en mutation.

\section{Conclusion}

Au terme de cette enquête, le dessin est apparu comme un matériau simple à recueillir et comme un outil riche d'enseignement, qui invite à poursuivre l'élaboration d'une méthodologie. Son avantage concret est de donner à voir, faire parler et vivifier les échanges autour de l'image ${ }^{10}$.

Le dessin a permis de saisir, accompagné des mots et des témoignages, l'absence de lien, pour les élèves résidant dans les zones frontalières du Nord, entre leur expérience de la frontière, dans la forme de l'interface, et l'idée qu'ils s'en font. Les élèves rencontrent au regard de notre enquête, une sorte d'obstacle épistémologique à penser 
ainsi l'ambivalence de la frontière, butant sur une définition scolaire figée dans la ligne séparatrice. Les dessins 1 à 4 rappellent cette définition de la ligne, le dessin 5 venant appuyer l'idée que la nature constitue un atout commode pour définir la frontière.

Sur le plan pédagogique, il conviendrait de faire du local un véritable terrain scientifique adapté aux élèves, puisqu'ils en sont déjà riches. Cela permettrait de leur montrer la complexité des dynamiques spatiales sur la frontière (coopération transfrontalière, développement des politiques culturelles dans l'Eurorégion, coordination policière, médicale...) dont ils sont déjà des témoins et des acteurs. Le local pourra donc ensuite servir pour mieux saisir le sens et le rôle des frontières opaques et fermées à la limite de l'Europe et ailleurs dans le monde.

Il serait intéressant de comparer cette distance entre représentations et témoignages observée lors de cette enquête dans le Nord avec d'autres terrains frontaliers en France. Là où la porosité est manifeste dans la forme du continuum (Zone Est), mais aussi avec des zones où les échanges avec l'autre côté de la frontière sont développés bien que les discontinuités spatiales physiques (Alpes, Pyrénées, Mer Méditerranée) soient un facteur limitant, et enfin, avec des régions qui ne sont pas limitrophes. Des études comparatives pourraient ainsi permettre de construire un gradient dessinant le sens des frontières et ses nuances, dans la géographie des enfants.

\section{BIBLIOGRAPHIE}

André Y., 1994. Du bon usage didactique des représentations spatiales. Revue de Géographie de Lyon, $\mathrm{n}^{\circ}$ 69, 3/94, p. 229-232.

André Y., 1989. Cartes mentales pour un territoire : à propos du Bassin de Genève. Mappemonde, n 89/1, p. 12-15.

André Y., Bailly A., Clary M. (dir), 1989. Représenter l'espace, l'imaginaire spatial à l'école. Paris, Antrophos-Economica.

Bailly A. et alii, 1995. Géographie régionale et représentations. Economica.

Bailly A., 1990. Paysages et représentations. Mappemonde, nº 90/3, p. 10-13.

Crozat D., 2001. Bar, boîtes et pralines. La permanente reconstruction culturelle d'une frontière entre Lille, la Belgique et le nord de l'Europe. Hommes et Terres du Nord, n 4, p. 230-241.

Danic I., Delalande J., Rayou P., 2006. Enquêter auprès d'enfants et de jeunes. Presses Universitaires de Rennes.

Gould P., White., 1984. Cartes mentales. Editions universitaires de Fribourg, Suisse.

Groupe Frontière, Arbaret-Schulz C., Beyer A., Piermay J.-L., Reitel B., Selimanovski C., Sohn C. et Zander P., 2004. La frontière, un objet spatial en mutation. EspacesTemps.net, Textuel, 29.10.2004, http://espacestemps.net/document842.html 
Gumuchian H., Marois C., Fevre V., 2000. Initiation à la recherche en géographie. Aménagement, développement territorial, environnement. Paris, Montréal, Economica, Presses de l'Université de Montréal.

Gumuchian H., 1981. La montagne française vue par l'enfant. Revue de Géographie Alpine, n 69, p. 35-68.

Hamez G., 2004. Du transfrontalier au transnational : approche géographique. L'exemple de la frontière franco-belge. Thèse de doctorat de géographie, Université Paris I.

Pagezy H., Carriere S. et Sabinot C., 2010. Nature du Monde, dessins d'enfants. CTHS.

Picouet P., Renard J-P., 2007. Les frontières mondiales. Editions du Temps.

Picouet P., 2011. Le monde vu à la frontière. L'Harmattan.

Renard J-P., 1997. Le géographe et les frontières. L'Harmattan.

Roumegous M., Clerc P., 2008. Le bon sens tout près de chez soi : les projets de programme de géographie pour l'enseignement primaire. Cybergeo, Les nouveaux programmes dans le primaire, mis en ligne le 03 octobre 2008, http://cybergeo.revues.org/index20383.html

Voye L., 1996. Le paysage, une géographie du sentiment légitime. Bulletin de la Société Géographique de Liège, $\mathrm{n}^{\circ}$ 32, 1996, p. 328-336.

\section{NOTES}

1. Programmes 2008 pour le cycle 3.

2. Nous remercions Mr Jean-Pierre Renard pour sa collaboration sur ces regards d'anciens manuels scolaires.

3. C'est à ce palier que la géographie fait son entrée à l'école car le cycle 2 (Grande Section, CP et CE1) doit, lui, structurer l'espace proche des élèves par une sorte de pré-géographie (comment se repérer dans la classe, l'école,...?, comment se représenter l'espace au moyen de photographies, maquettes, plans... ?) et faire découvrir, de manière plus générale, qu'ailleurs, c'est différent.

4. Des questionnements méthodologiques similaires ont été posés dans le cadre d'une rechercheaction de l'IUFM Nord-Pas-de-Calais: "Discontinuités spatiales et apprentissages scolaires ", 2008-2010, menée par Sylvie Considère et Nicolas Lebrun avec la participation de Xavier Leroux.

5. On entend par spiralaire le fait que le questionnaire se développe dans la logique d'une spirale : les objectifs des questions se déroulent au long de l'enquête sans suivre un fil linéaire, mais en faisant revenir, en quelque sorte, le questionnaire sur lui-même. C'est un moyen de limiter la capacité d'anticipation de certains enquêtés qui, comprenant les attentes de l'enquête, pourraient vouloir contrôler leur réponse et écrire ce qu'ils pensent être attendu.

6. Voir à ce titre le sujet du brevet des collèges 2011 qui invitait les collégiens à identifier des pays comme des pièces de puzzle placées hors de tout contexte, sans situation et surtout sans échelle faisant, par exemple, du Brésil un État insuffisamment vaste par rapport à l'Italie ou au Japon et que certains ont pu confondre avec l'Inde ou encore même l'Afrique: http:// asset.rue89.com/files/brevet_college_corse_italie_grand.jpg

7. Nous avons montré, par ce qui précède, qu'ils l'ont appris à l'école.

8. Picouet P., Renard J-P., 2007. Les frontières mondiales. Editions du Temps, p. 30.

9. On peut impliquer les élèves dans cette réflexion en partant de questions simples telles que : «Pourquoi paie-t-on ses frites en euro en Belgique? Comment faisait-on avant? Pourquoi ne donne-t-on pas ses papiers pour passer en Belgique mais les donne-t-on pour le Maroc ou à l'aéroport ? Pourquoi peut-on prendre un seul bus pour aller de Roubaix à Mouscron ? " 
10. Il apporte aussi un peu de fraïcheur dans la communication scientifique, impression partagée lors de leur présentation en séminaire interne au laboratoire Dynamiques des Réseaux et Territoires en mai 2010.

\section{RÉSUMÉS}

Quelle géographie de la frontière peut se faire un enfant de région frontalière ? L'article tente de montrer et de comprendre comment cet objet se construit dans la représentation d'enfants de 8 à 11 ans qui l'apprennent à l'école et qui, pour certains, la vivent au quotidien. Une enquête au sein de classes de cycle 3, menée dans le Nord de la France a permis de saisir ces représentations et de révéler le dessin comme outil méthodologique pertinent.

What king of geographical sense does a child make of a cross-border region? The article attempts to show and understand how the border is depicted in their mind, while school has to teach them what it is. Our survey, carried out in primary school classes in the cross-border region of North of France, collected these representations and attempts to show that drawing is a relevant methodological tool.

\section{INDEX}

Keywords : border, drawing, representation, story, teaching geography

Mots-clés : dessin, enseignement de la géographie, frontière, représentation, témoignage

\section{AUTEURS}

\section{XAVIER LEROUX}

Xavier Leroux, xavleroux@free.fr, est Professeur des écoles, chercheur associé au laboratoire Dynamique des Réseaux et Territoires, Université d'Artois. Il a publié :

- Leroux X., 2010. Logiques d'affectation des professeurs des écoles néotitulaires dans le Nord. Cybergeo : European Journal of Geography [En ligne], Epistémologie, Histoire de la Géographie, Didactique, article 488, mis en ligne le 10 février 2010, URL : http://cybergeo.revues.org/22918 ; DOI : $10.4000 /$ cybergeo.22918

\section{MAUD VERHERVE}

Maud Verherve, mverherve@gmail.com, est Professeur des écoles, doctorante au laboratoire Territoires, Villes Environnement et Société, Université de Lille 1. Elle a publié récemment : - Verherve M., 2012. Entreprendre de savoir comment et jusqu'où il serait possible de penser autrement l'espace. Materiali Foucaultiani, volume I, numéro 1 (janvier-juin 2012), ISSN 2239-5962, http://www.materialifoucaultiani.org/it/rivista/ultimo-numero.html 